\title{
4 Kurznachrichtenkommunikation
}

Die vorliegende Arbeit beschäftigt sich - wie in der Einleitung angekündigt mit der SMS-Kommunikation sowie mit der Kommunikation, die über die internetbasierten Messenger-Dienste WhatsApp, Viber und iMessage erfolgt. Die zwei folgenden Unterkapitel stellen den aktuellen Forschungsstand zur elektronischen Kurznachrichtenkommunikation per SMS, WhatsApp, Viber und iMessage kurz dar, wobei in erster Linie auf linguistische Arbeiten zum Deutschen und Russischen eingegangen wird.

\subsection{SMS-Kommunikation}

Die SMS-Kommunikation ist Anfang der 2000er in den Fokus (sozio)linguistischer Forschung gerückt. $\mathrm{Zu}$ den ersten empirischen Arbeiten zur deutschen SMS-Kommunikation ${ }^{64}$ zählen die von Schlobinski et al. (2001) sowie die von Androutsopoulos/Schmidt (2002) ${ }^{65}$ (vgl. Dürscheid/Frick 2014: 150-152). Einige Jahre später erschienen die ersten Studien zur russischsprachigen SMS-Kommunikation (vgl. Nikitin/Avdonina 2005, 2006; Dremina 2006; Sidorova 2006). ${ }^{66}$

Aus theoretischer Perspektive wird die SMS-Kommunikation in zahlreichen deutschsprachigen Arbeiten in Anlehnung an Dürscheid (2005) sowie - v.a. in früheren Untersuchungen - an Holly (1997) als Kommunikationsform betrachtet

64 Die SMS-Kommunikation ist außerdem zum Gegenstand mehrerer englischsprachiger Untersuchungen geworden. Darunter sind v.a. die Arbeiten von Thurlow (2003), Laursen (2005), Ling/Baron (2007, 2013), Baron (2008), Frehner (2008), Tagg (2009), Herring/Stein/Virtanen (2013) und Thurlow/Poff (2013) zu nennen.

65 Die im Netz zur Verfügung gestellte Preprint-Version (http://www.ids-mannheim.de/prag/ sprachvariation/tp/tp7/SMS-Kommunikation.pdf) stammt jedoch aus dem Jahr 2001 (vgl. dazu das Literaturverzeichnis in Schlobinski et al. 2001 sowie Dürscheid/Frick 2014: 157).

66 Die Tatsache, dass die ersten Beiträge zur russischen SMS-Kommunikation vier bis fünf Jahre später als diejenigen zur deutschen erschienen sind, lässt sich vermutlich auf die historische Entwicklung der Mobilfunkdienste in Russland zurückführen. Laut der Angaben auf der Internetseite des russischen Mobilfunknetzbetreibers МегаФон wurde der SMS-Versand als Dienstleistung zum ersten Mal im Jahr 1996 angeboten (vgl. http://corp.megafon.com/about/ history/). El'bert (2012: 245) merkt an, dass alle Mobilfunknetzbetreiber bis zum Jahr 2000 das Verschicken von SMS-Nachrichten innerhalb ihrer eigenen Mobilfunknetze ermöglicht haben, während der Nachrichtenaustausch zwischen Kunden unterschiedlicher Mobilfunknetzbetreiber erst seit 2002 möglich geworden ist. Dies trägt u.a. dazu bei, dass Mobilfunkdienste in Russland seit Anfang der 2000er immer öfter in Anspruch genommen wurden und die Anzahl der Kunden stetig anstieg (vgl. Savel'ev 2011: 94). 
(vgl. Androutsopoulos/Schmidt 2002; Imo 2012; König 2015a). In der russischen Forschungstradition wird die SMS-Kommunikation als тип дискурса (,Diskurstyp', vgl. El'bert 2012), жанр (,Genre', vgl. Sidorova 2006) oder форма общения (,Kommunikationsform', vgl. Venediktova 2011) aufgefasst, wobei die damit bezeichneten theoretischen Konzepte eine Reihe von Überschneidungen bzw. Berührungspunkte aufweisen (für eine ausführliche terminologische Diskussion vgl. Kushakova 2015: 15-41). In der vorliegenden Untersuchung wird in Bezug auf die SMS-Kommunikation in beiden Sprachen der Begriff Kommunikationsform verwendet, der sich inzwischen in den einschlägigen Arbeiten zur SMS-Kommunikation im deutschsprachigen Raum etabliert hat.

Als Kommunikationsform lässt sich die SMS-Kommunikation nach den von Dürscheid (2005) herausgearbeiteten Kriterien als schriftlich realisierte, (prototypisch) dialogisch ausgerichtete Kommunikation, die sich i.d.R. zwischen zwei Personen vollzieht, beschreiben. In der Rolle des Kommunikationsmediums tritt dabei ein Mobiltelefon bzw. ein Smartphone auf. Die Kommunikation findet i.d.R. unter Bedingungen der räumlichen Distanz statt, während hinsichtlich der zeitlichen Dimension in der Forschungsliteratur kein Konsens besteht: Die SMS-Kommunikation wird als quasi-synchron oder asynchron eingeordnet, was v.a. durch die zeitlichen Abstände zwischen einzelnen Nachrichten bedingt ist (vgl. dazu Venediktova 2011: 57, 70-76; Imo 2012: 22-23, 2015a: 7; König 2015a: 90-92; zum graduellen Charakter der Quasi-Synchronizität vgl. Dürscheid 2016).

Nachdem die SMS-Kommunikation als Kommunikationsform definiert wurde, soll nun ein Einblick in die sprachlichen Phänomene und nonverbalen Mittel gegeben werden, die für die russische und deutsche SMS-Kommunikation belegt sind. Dabei werden für die Darstellung einzelner ausgewählter Phänomene der SMS-Kommunikation der jeweiligen Sprache die von Androutsopoulos (2007: 81-84) herausgearbeiteten Kategorien der ,neuen' Schriftlichkeit herangezogen: (i) Phänomene konzeptioneller Mündlichkeit, (ii) mimisch-kinesische Kompensierungsverfahren, (iii) graphostilistische Ressourcen und (iv) Verfahren sprachlicher Ökonomie. In Bezug auf einzelne verbale und nonverbale Mittel lässt sich anhand von Ergebnissen empirischer Studien zur SMS-Kommunikation in der jeweiligen Sprache erkennen, dass russische und deutsche SMS-Dialoge die gleichen bzw. ähnlichen Phänomene aufweisen. Abgesehen von der vorliegenden Untersuchung sowie einzelnen Arbeiten, die auf der Basis der einschlägigen Forschungsliteratur auf ausgewählte Phänomene in der SMS-Kommunikation in den beiden Sprachen eingehen (vgl. Palkova 2011), 
bestehen $\mathrm{m} . \mathrm{W}$. jedoch keine systematischen sprachvergleichenden ${ }^{67}$ empirischen Studien zur deutschen und russischen SMS-Kommunikation. ${ }^{68}$

Im Bereich der Phänomene konzeptioneller Mündlichkeit zeichnet sich die SMS-Kommunikation im Russischen und Deutschen z.B. durch die Schreibungen aus, die die gesprochensprachliche lautliche Gestaltung eines Wortes wiedergeben, sodass Phänomene wie Tilgungen, Klitisierungen und Reduktionen verschriftlicht werden (vgl. Androutsopoulos 2007: 82 sowie „mündliche Schreibung“ bei Androutsopoulos/Ziegler 2003). Dabei sind u.a. hab und hastefür das Deutsche oder die Reduktion im Wortinlaut von „скоко“ (für сколько, wie viel') für das Russische (vgl. Venediktova 2011: 209) zu nennen. Das Gleiche gilt auch für die graphische Darstellung emulierter Prosodie (vgl. Androutsopoulos 2007: 82 sowie Haase et al. 1997), die in den beiden Sprachen u.a. durch Majuskelschreibung (z.B. NEIN! für das Schreien) oder Zeicheniterationen (z.B. Jaaaa! für die Lautdehnung) zum Ausdruck gebracht werden kann (vgl. dazu Schlobinski et al. 2001; Savel'ev 2011; Venediktova 2011; Schnitzer 2012).

$\mathrm{Zu}$ mimisch-kinesischen Kompensierungsverfahren im Sinne von Androutsopoulos (2007: 82) gehört der Gebrauch von Emoticons (vgl. Schlobinski et al. 2001; Schnitzer 2012; Imo 2015b für das Deutsche sowie Sidorova 2006; Savel'ev 2011; Venediktova 2011; Kushakova 2015 für das Russische) sowie von Aktionswörtern (vgl. Bartz/Beißwenger/Storrer 2014). Als Aktionswörter fungieren im Deutschen in erster Linie Inflektive ( $\left.{ }^{*} l a c h{ }^{\star}\right)$ bzw. Inflektivkonstruktionen ( ${ }^{*}$ liebguck ${ }^{\star}$ ) und Akronyme $\left({ }^{\star} g^{\star}{ }^{\star}\right.$, wobei deren Gebrauch in der SMS-Kommunikation eine deutlich niedrige Frequenz als in der Kommunikation via Internet Relay Chat aufweist (vgl. Schlobinski et al. 2001: 14; Schnitzer 2012: 141-143). In der russischen SMS-Kommunikation ist die Verwendung von Aktionswörtern in SMS-Nachrichten ebenfalls relativ selten belegt. Dabei fällt allerdings auf, dass im Gegensatz zum Deutschen russische Aktionswörter meist als finite Verbformen realisiert werden, z.B. als Verben der 1.P. Sg. („привет!/улыбаюсь/“ -

67 Eine sprachkontrastive Untersuchung der deutschen und russischen SMS-Kommunikation steht zwar noch aus, es liegen jedoch bereits Arbeiten vor, in denen die SMS-Kommunikation im Russischen oder Deutschen mit derjenigen einer anderen Sprache verglichen wird (für die SMS-Kommunikation im Russischen und Englischen vgl. Venediktova 2011; für die SMS-Kommunikation im Deutschen und Englischen vgl. Bieswanger 2007; für die SMS-Kommunikation im Deutschen und Chinesischen vgl. Günthner 2011, 2014; Günthner/Kriese 2012; Hauptstock/König/Zhu 2010 sowie Imo 2012; für die SMS-Kommunikation im Deutschen und Japanischen vgl. Hayashi/Imo/Ogasarawa 2020 und Schlobinski/Watanabe 2003).

68 Meines Wissens existiert bisher lediglich eine einzige Untersuchung von Palkova (2011), die im Sinne einer Metastudie versucht, Gemeinsamkeiten und Unterschiede in der SMS-Kommunikation in den beiden Sprachen aufzuzeigen. 
,hallo!/(ich) lächle/‘ aus Venediktova 2011: 108). Wie das Beispiel zeigt, werden Aktionswörter von russischsprachigen Schreibern etwa mit Schrägstrichen umklammert, wobei - zumindest für die Chat-Kommunikation via Internet Relay Chat - auch die Asteriskmarkierung belegt wurde (vgl. Gokh 2011; Arzhantseva 2012). Die Verwendung von Emoticons scheint in den beiden Sprachen formal und funktional eine Reihe von Überschneidungen aufzuweisen, wobei ein genauer Blick auf deren Häufigkeit und potenzielle Divergenzen beim Gebrauch nötig ist. Aus formaler Perspektive soll allerdings auf eine für das Russische besondere Form der Emoticons :-) und :-(hingewiesen werden: Die beiden können lediglich auf einen ihrer ,Bestandteile', nämlich auf die den Mund symbolisierende Klammer, reduziert werden und als ) bzw. (auftreten (vgl. Savel'ev 2011: 69; Venediktova 2011: 115). Für das Deutsche ist dies nicht belegt. Eine quantitative Auswertung des Gebrauchs der Emoticon-Varianten :-), :) sowie ) anhand der russischen SMS-Nachrichten aus der Mobile Communication Database zeigt, dass die reduzierte Variante ${ }^{69}$ mit 1.210 Tokens mehr als drei Mal so oft verwendet wird wie :-)(142 Tokens) und :)(242 Tokens).

Des Weiteren finden sich in der SMS-Kommunikation sowohl im Deutschen als auch im Russischen graphostilistische Schreibungen (vgl. Androutsopoulos 2007: 83) wie „Gute N8“ (Schnitzer 2012: 527) oder „05“ (Venediktova 2011: 203: опять ,wieder', das sich aus $0+$ пять, fünf‘ zusammensetzt).

Auch bei den Ökonomisierungsstrategien lassen sich gewisse Parallelen zwischen deutschen und russischen SMS-Nachrichten erkennen: Es tauchen Kurzwörter und Abkürzungen, Ellipsen, das Weglassen von Satz- und Leerzeichen und die Binnenmajuskelschreibungen u.Ä. auf (vgl. Schlobinski et al. 2001; Sidorova 2006; Venediktova 2011: 211-212; Schnitzer 2012). Solche Schreibstrategien, „die von technisch-medialen Einschränkungen gefördert bzw. geradezu erzwungen werden“ (Androutsopoulos 2007: 83), werden im Falle der SMS-Kommunikation v.a. mit der pro Nachricht geltenden Zeichenbegrenzung $^{70}$ in Verbindung gebracht, die sich für eine auf Deutsch verfasste

69 Diese Auswertung berücksichtigt auch die iterierten Varianten aller drei Emoticons.

70 Es ist anzumerken, dass die Zeichenbegrenzung in der Forschungsliteratur zur SMS-Kommunikation in den beiden Sprachen häufig als Hauptargument für das Vorkommen von sprachlichen Mitteln, die als sprachökonomisch gelten, genannt wird. Laut einer Reihe von empirisch basierten Studien liegt die durchschnittliche Länge einer SMS-Nachricht jedoch meist unter der maximalen Zeichenanzahl (je nach Geschlecht, Bildungsgrad und Alter zwischen 32,8 und 103,7 Zeichen pro Nachricht in den Daten von Schnitzer (2012: 47-48) bzw. bei ca. 110 Zeichen pro Nachricht im Schweizer SMS-Korpus, vgl. Dürscheid/Frick (2014: 169)). Daher muss mit dem Argument der durch die Zeichenbegrenzung ausgelösten Ökonomisierung entsprechend vorsichtig umgegangen werden. 
SMS-Nachricht auf 160 Zeichen und für eine russische SMS-Nachricht auf lediglich 70 Zeichen ${ }^{71}$ beläuft. In diesem Zusammenhang stellt sich die Frage, ob eine solch markante Differenz bei der Zeichenbeschränkung in der jeweiligen Sprache Divergenzen beim Schreibverhalten auslöst (vgl. dazu die Rolle von Affordanzen für das sprachliche Handeln in der interaktionalen Schriftlichkeit bei Hutchby 2001 und Zillien 2009). Anders formuliert: Führt eine maximale Nachrichtenlänge von 70 Zeichen (v.a. in der Anfangsphase, in der SMS-Nachrichten per Einzelabrechnung abgerechnet wurden) dazu, dass russische Schreiber stärker auf unterschiedliche Ökonomisierungsverfahren angewiesen sind bzw. dass sie bestimmte Schreibstrategien entwickeln und anwenden müssen, um die für das Russische ,ungerechte‘ Zeichenbeschränkung zu umgehen? In der Tat wird in der Forschungsliteratur zur russischen SMS-Kommunikation ein Phänomen als eine systematische Ökonomisierungsstrategie angesehen: Dabei geht es um die Schreibstrategie, die bereits in der informellen schriftbasierten Kommunikation in Internet Relay Chats, Foren und via E-Mails angewendet wurde und darin besteht, das Russische mithilfe von lateinischen Buchstaben, Ziffern und Sonderzeichen darzustellen, indem man einerseits auf die herkömmlichen Regeln der Transkription und Transliteration zurückgreift, anderseits aber idiosynkratische visuell oder anders motivierte Zuordnungen schafft (vgl. Venediktova 2011: 117-120; El'bert 2012). Die Entstehung dieses Phänomens, das den Namen транслит (,Translit') - eine Abkürzung des Nomens транслитерация (,Transliteration') - trägt, wurde durch die technische Beschaffenheit der ersten für die schriftbasierte computervermittelte Kommunikation $^{72}$ eingesetzten Medien bedingt (vgl. Hutchby 2001; Zillien 2009 und Marx/Schmidt 2019 zum Konzept Affordanzen): Die ersten PCs - und später auch die ersten auf den Markt gekommenen Handys - waren weder mit der entsprechenden Tastatur ausgestattet, noch waren sie im Stande, den russischen Text zu kodieren und zu dekodieren, sodass man gezwungen war, mit

71 Dieser große Unterschied in der Zeichenanzahl pro Nachricht, die in den beiden Sprachen den gleichen maximalen Umfang von 140 Bytes (=1.120 Bits) hat, ergibt sich aus der Kodierung lateinischer und kyrillischer Buchstaben. Während ein lateinischer Buchstabe - ebenso wie eine arabische Ziffer - mit lediglich 7 Bits kodiert wird (1.120 Bits : 7 Bits $=160$ Zeichen), benötigt man zum Kodieren eines kyrillischen Buchstabens mehr als doppelt so viele Bits, nämlich 16, sodass eine in kyrillischer Schrift geschriebene Nachricht bei ausgeschöpfter maximaler Zeichenanzahl weniger als halb so lang wie eine entsprechende deutsche ist (1.120 Bits : 16 Bits $=70$ Zeichen).

72 In der vorliegenden Arbeit wird der etablierte Begriff computervermittelte Kommunikation verwendet (zur Diskussion der weiteren Begriffe v.a. des von Jucker/Dürscheid (2012) vorgeschlagenen Konzepts Keyboard-to-Screen-Kommunikation vgl. Imo 2015a). 
lateinischen Buchstaben auszukommen. ${ }^{73}$ Somit besteht in der russischen SMSKommunikation eine „computer-mediated digraphia“ im Sinne von Androutsopoulos (2009: 222), indem zwei Schriftsysteme - das kyrillische und das lateinische - gleichzeitig angewendet werden können. Bei der Anwendung des Translit-Schreibens in der SMS-Kommunikation trat v.a. die Kostenfrage in den Vordergrund: ${ }^{74}$ Man kann den Text, der in kyrillischer Schrift zwei Nachrichten in Anspruch nehmen würde, in einer Nachricht verschicken und somit Geld sparen. Darüber hinaus schreibt Venediktova (2011: 118) dem Translit eine Selbstdarstellungsfunktion zu: Wer Nachrichten in Translit verfassen und lesen kann, gilt als erfahrener SMS-Nutzer, da die Produktion sowie die Rezeption einer in Translit geschriebenen Nachricht tatsächlich kein leichtes Unterfangen ist. Der Grund dafür ist, dass für das Translit - ähnlich wie für das von Androutsopoulos (2009) beschriebene Latin-alphabet Greek - kein einheitliches Regelsystem für die Wiedergabe russischer Buchstaben vorliegt, sondern die Zuordnung mit einem mehr oder weniger geringen Grad der Beachtung der offiziellen Regeln für die Transkription bzw. die Transliteration individuell erfolgt, was zur Entstehung idiosynkratischer - manchmal äußerst kreativer - Translitsysteme führt. Das russische $c[\mathrm{~s}]$ kann beispielsweise mit dem lateinischen $c$ (graphische Übereinstimmung) oder mit dem lateinischen $s$ [s] (phonetische Übereinstimmung) dargestellt werden. Bei $\phi$ [f] stößt man neben dem herkömmlichen phonetisch motivierten $f[\mathrm{f}]$ auch auf die kreative, an die optische Ähnlichkeit angelehnte Variante ${ }^{o} I^{o}$ (Venediktova 2011: 123). Die auf diese Weise entstehende Variabilität des Translit-Schreibens - es wurden beispielsweise bis zu 23 Verschriftungsmöglichkeiten für den russischen Buchstaben щ [t $\left.\oint^{\dagger}\right]$ verzeichnet (vgl. Venediktova 2011: 125) -, die das Schreiben, das Lesen sowie das Verstehen der SMS-Nachrichten teilweise beeinträchtigen kann, hat in Kombination mit der technischen Entwicklung der Mobiltelefone und der Verringerung der Kosten für den SMS-Versand zu einem Rücklauf der Translit-Nutzung geführt. Während im Korpus von Savel'ev (2011: 62) (1.315 SMS-Nachrichten, erhoben von 2004 bis 2010) 80\% der Nachrichten in Translit verfasst wurden, beträgt

73 Analoge Verfahren des Schriftsystemwechsels, auch script-switching genannt (vgl. Androutsopoulos 2009: 228 sowie Ma i.Dr.), sind auch für das Griechische (vgl. „Greeklish“ bzw. „Latin-alphabet Greek“ bei Androutsopoulos 2009: 221) sowie das Arabische (vgl. „Arabizi“ bei Palfreyman/Al Khalil 2003) beschrieben worden.

74 Die technisch bedingte Motivation beim Translit-Schreiben spielt zwar bei der SMS-Kommunikation nicht mehr die zentrale Rolle, sie bleibt dennoch für Schreiber aktuell, die über ein Handy mit einer Zahlentastatur ohne Belegung mit kyrillischen Buchstaben und/oder ohne entsprechende Spracheinstellungen verfügen. Für einen durch ein Dekodierungsproblem ausgelösten Wechsel zum Translit vgl. (202). 
deren Anteil im Korpus von Venediktova (2011) (759 SMS-Nachrichten, erhoben von 2008 bis 2009) lediglich 32\%. Im russischen Teilkorpus der MoCoDa (erhoben von 2013 bis 2015) machen 215 in Translit verfasste SMS-Nachrichten lediglich ca. 5\% des gesamten Korpus aus, wobei die Translit-Schreibung dabei in erster Linie durch die technische Beschaffenheit des jeweiligen Eingabegeräts (also durch das Vorhandensein einer physischen Tastatur, die nur mit lateinischen Buchstaben belegt ist) und nur in Einzelfällen durch die Kostenminimierung motiviert war. Einen besonderen Fall stellt die Verwendung des Translits beim sogenannten code-switching ohne script-switching (vgl. Ma i.Dr.) dar, bei dem nach einem Code-Switching vom Russischen ins Englische und wieder zurück die Tastatursprache absichtlich nicht wieder vom Englischen aufs Russische zurückgestellt wird (vgl. dazu \#2632 in der MoCoDa).

In Hinblick auf die methodische Vorgehensweise sowie die Sicht auf die Daten lassen sich die meisten der oben erwähnten Arbeiten zur SMS-Kommunikation laut König/Bahlo (2014: 5) sowie König (2015a: 88-89) als diejenigen einordnen, die sich mit ausgewählten sprachlichen Phänomenen innerhalb von isoliert betrachteten SMS-Nachrichten befassen, ohne ihre sequenzielle Einbettung in den Dialog zu berücksichtigen (vgl. Schlobinski et al. 2001; Schnitzer 2012 für das Deutsche sowie die Mehrheit der russischsprachigen Studien wie Savel'ev 2011; Venediktova $2011^{75}$ und Sirotkina 2012). Andere Studien hingegen legen einen großen Wert darauf, vollständige SMS-Dialoge aus gesprächsanalytischer Perspektive ${ }^{76} \mathrm{zu}$ analysieren (vgl. König 2015a: 88-89; König/Bahlo 2014: 5). Dieser Gruppe an Untersuchungen können etwa Arbeiten von Günthner (2011), Imo (2012, 2015a) oder König (2015a, 2015b) zugerechnet werden. Dabei wird mit Methoden und Konzepten der Konversationsanalyse und der Interaktionalen Linguistik zum einen die dialogische Organisation der SMS-Kommunikation als solche aufgezeigt, zum anderen wird eine sequenzielle Analyse unterschiedlicher kommunikativer Gattungen (vgl. Luckmann 1986, 1988; Günthner/Knoblauch 1994, 1996; Günthner 1995, 2006) durchgeführt, die sich innerhalb der SMS-Kommunikation herausgebildet haben. So zeigt Günthner

75 Die starke Orientierung an der Analyse isolierter Nachrichten im Gegensatz zu zusammenhängenden Dialogen lässt sich in der Dissertation von Venediktova (2011) bereits in der Phase der Datenerhebung erkennen: In einem dafür eingesetzten Fragebogen wurden ,Datenspender darum gebeten, insgesamt sechs SMS-Nachrichten für Forschungszwecke zur Verfügung zu stellen, und zwar die letzten drei von ihnen verschickten sowie die letzten drei von ihnen empfangenen Nachrichten.

76 Die Frage, ob und inwiefern die für die Analyse gesprochensprachlicher Daten entwickelten Methoden der Gesprächsanalyse sowie der Interaktionalen Linguistik für deren Anwendung bei der Untersuchung der SMS-Daten geeignet sind, wird in Kap. 5 diskutiert. 
(2011), dass ein per SMS erfolgter Nachrichtenaustausch mit einer Begrüßung als Einstiegssequenz und einer Verabschiedung als Beendigungssequenz gerahmt werden kann. Des Weiteren sind SMS-Nachrichten innerhalb eines Dialogs laut Günthner (2011) sequenziell organisiert, sie bilden Paarsequenzen, sodass eine Nachricht als first pair part eines Adjazenzpaares eine bestimmte Reaktion des Interaktionspartners erwartbar macht, wobei bei der Gestaltung einzelner Beiträge das Rezipientendesign zum Tragen kommt (vgl. dazu auch Günthner/Kriese 2012; König 2015a). Der Frage, wie sich „das Prinzip der Dialogizität“ (Günthner 2011: 7) in der sequenziellen Organisation verschiedener kommunikativer Gattungen der SMS-Kommunikation niederschlägt, wird beispielsweise für SMS-Verabredungen bei Hauptstock/König/Zhu (2010) und für die Liebeskommunikation bei Imo (2012) nachgegangen. Für weitere gesprächsanalytisch ausgerichtete Arbeiten zur SMS-Kommunikation sei auf den Sammelband von König/Bahlo (2014) verwiesen. Zur russischen SMS-Kommunikation sind mir keine Studien bekannt, die eine gesprächsanalytische Perspektive auf die Daten vertreten.

\subsection{Kommunikation via WhatsApp, Viberund iMessage}

Unter internetbasierten Messenger-Diensten, die in den letzten Jahren einen festen Platz in unserem Alltag eingenommen haben, gilt WhatsApp laut statistischen Berichten für 2018 sowohl in Deutschland (81\% der Befragten) ${ }^{77}$ als auch in Russland (58\% der Befragten) $)^{78}$ als die meist genutzte Messenger-App. Die zwei weiteren Messenger-Dienste Viber und iMessage, die für die vorliegende Arbeit relevant sind, sind vergleichsmäßig weniger beliebt. 10\% der Informanten in Deutschland gaben an, dass sie iMessage benutzen, während Viberin der Statistik nicht aufgeführt wird. ${ }^{79}$ In Russland dagegen stellt Viber mit 38\% den zweitpopulärsten Messenger-Dienst dar, während Angaben zur iMessage Nutzung fehlen. ${ }^{80}$

77 Die Umfrage, an der 1.212 Informanten ab einem Alter von 14 Jahren teilgenommen haben, wurde vom Bundesverband Informationswirtschaft, Telekommunikation und neue Medien e.V. Bitkom durchgeführt (vgl. https://t1p.de/flpy).

78 Die Angabe stammt aus dem Bericht des Deloitte CIS Research Centre, das 2018 eine Umfrage unter 1.600 Respondenten im Alter zwischen 18 und 55 Jahren aus unterschiedlichen Regionen Russlands zu deren Mediennutzungsverhalten durchgeführt hat (vgl. https://t1p.de/igdq). 79 Vgl. https://t1p.de/flpy.

80 Vgl. Fußnote 79. 
Die statistischen Werte spiegeln sich in der linguistischen Forschung wider - während die WhatsApp-Kommunikation (v.a. im deutschsprachigen Raum) in den Fokus einer Reihe von linguistischen Arbeiten gerückt ist, genießen die Viber- sowie iMessage-Kommunikation eine wesentlich geringere Aufmerksamkeit. Studien zur WhatsApp-Kommunikation werden zudem zusätzlich durch den Aufbau von WhatsApp-Datenkorpora begünstigt, wie etwa der Datenbank Mobile Communication Database 2 (https://db.mocoda2.de) sowie der beiden im Rahmen der Projekte What's up, Switzerland? (Datenerhebung im Sommer 2014) und What's up Deutschland? (Datenerhebung im Winter 20142015) erhobenen Korpora, die nach dem Ablauf der Projektfristen ebenso für Forschungs- und Lehrzwecke zur Verfügung gestellt werden.

Im Gegensatz zur SMS-Kommunikation, die lediglich den Austausch von Textnachrichten ermöglicht, bietet WhatsApp als internetbasierter Messenger eine breitere Palette an Kommunikationsressourcen an: Neben Textnachrichten, die sowohl im Rahmen von dyadischen als auch Gruppenchats ausgetauscht werden können, können auch Bilder, Videos, Audios, Links sowie Standortinformationen verschickt werden (vgl. Arens 2014; Dürscheid/Frick 2014). Zudem wird bei der WhatsApp-Installation für jeden Nutzer ein Profil hinterlegt, der mit einem Profilbild sowie einer ergänzenden Information ausgestattet werden kann (vgl. König/Hector 2017: 8). Des Weiteren kann analog zu Facebook ein Profilstatus eingegeben werden (vgl. König/Hector 2017: 8). Außerdem ist auch die Möglichkeit gegeben, über die Internetverbindung geleitete Audio- und Video-Anrufe zu tätigen. Eine solche Überlagerung mehrerer einem WhatsAppNutzer zur Verfügung stehender Kommunikationskanäle sowie -ressourcen (vgl. „formal-funktionale Hybridität“ im Sinne von Wyss/Hug 2016: 259) führt dazu, dass WhatsApp nicht mehr als eine Kommunikationsform angesehen werden kann, sondern inzwischen als ,eine modular aufgebaute internetbasierte Kommunikationsplattform“ (König/Hector 2017: 7) aufgefasst wird (vgl. auch dazu Dürscheid/Frick 2014"; Wyss/Hug 2016; Frick 2017). Dabei plädieren König/Hector (2017: 10) dafür, innerhalb der Kommunikationsplattform WhatsApp einzelne Kommunikationsformen $\mathrm{zu}$ unterscheiden wie „Statusmitteilungen, Statusinformationen, Gruppenchats, dyadische Chats, Sprach- und Videotelefonie“. Dürscheid/Frick (2014: 166) sind ebenso der Meinung, dass man auf-

81 Es sei hier angemerkt, dass Dürscheid/Frick (2014: 152) zwar ebenso darauf hinweisen, dass WhatsApp eigentlich eine Kommunikationsplattform darstellt, verwenden jedoch ,aus Kompatibilitätsgründen“ den Begriff Kommunikationsform in Anlehnung an Dürscheid (2005), welchen sie allerdings erweitern und sowohl einzelne Kommunikationsformen wie SMS als auch Kommunikationsplattformen wie WhatsApp darunter fassen. 
grund des Zeichensystems Sprachnachrichten (Zeichensystem: gesprochene Sprache) von Textnachrichten (Zeichensystem: geschriebene Sprache) unterscheiden und die beiden daher als zwei eigenständige Kommunikationsformen auffassen sollte. An dieser Stelle stellt sich aber die Frage, ob der Versand von Bildern und Videos in diesem Fall nicht ebenso als eigene Kommunikationsform angesehen werden muss (zur Multimodalität in der WhatsApp-Kommunikation im Deutschen vgl. Arens 2014; eine umfragebasierte soziolinguistische Untersuchung zur Verteilung der multimedialen Ressourcen in der WhatsAppKommunikation im Russischen findet sich in Balakina/Sosnin 2017).

Am besten ist bisher die Kommunikation über die Chat-Funktion - und zwar v.a. in der dyadischen Konstellation - untersucht (vgl. Imo 2015a; König 2015a; Bauer 2016; Pappert 2017; Mostovaia 2018, 2021), während andere von König/Hector (2017) thematisierte Kommunikationsformen noch kaum erforscht sind (vgl. aber die soziolinguistische Studie zu Statusinformationen bei Sánchez-Moya/Cruz-Moya 2015 sowie König/Hector 2017: 8-9). Zu Sprachnachrichten liegen bislang einige wenige Arbeiten vor, die datenbasiert (vgl. Hector 2017; König/Hector 2017) sowie umfragebasiert (vgl. Orlova 2018) vorgehen.

Noch eine technische Neuerung betrifft den Umfang einer schriftbasierten WhatsApp-Nachricht, die sich für das Deutsche momentan auf 65.536 Zeichen beläuft (zur Zeichenbegrenzung für deutsche und russische SMS-Nachrichten jeweils 160 und 70 Zeichen pro Nachricht vgl. Kap. 4.1). Für die maximale Länge einer russischen WhatsApp-Nachricht konnten leider keine Informationen gefunden werden, eine Reihe von selbst durchgeführten Tests hat aber gezeigt, dass je nachdem, ob eine Textnachricht neben Buchstaben des kyrillischen Alphabets Zahlen und/oder Interpunktionszeichen enthält, die gesamte Nachrichtenlänge zwischen 65.350 und 65.400 Zeichen schwanken kann. Diese erheblich höhere Zeichengrenze mag zwar die Annahme nahelegen, dass WhatsApp-Nachrichten auch entsprechend länger als SMS-Nachrichten sind. Dies entspricht jedoch der - zumindest für das Deutsche nachgewiesenen Tendenz zum Verschicken von kurzen WhatsApp-Nachrichten nicht (vgl. Dürscheid/Frick 2014: 169; Imo 2015a sowie Wyss/Hug 2016). ${ }^{82}$ Imo (2015a: 15) spricht in diesem Zusammenhang von der in der WhatsApp-Kommunikation beobachtbaren „Präferenz, für jede Einzelhandlung eine eigene Nachricht zu

82 Dürscheid (2016: 456) berichtet, dass WhatsApp-Nachrichten aus dem Korpus des Projekts What's up Switzerland? (838.238 Nachrichten) im Durchschnitt lediglich 30 Zeichen lang sind, wobei dieser relativ geringe Durchschnittswert v.a. durch eine hohe Anzahl an Nachrichten zustande kommt, die nur ein Wort oder ein Emoji enthalten und dadurch die Statistik möglicherweise etwas verzerren. 
verwenden“, während eine SMS-Nachricht dagegen prototypischerweise aus mehreren Einzelhandlungen besteht (vgl. dazu auch König 2015a: 99-100). Des Weiteren zieht Imo (2015a: 15, 22-24) Parallelen zwischen der schriftbasierten WhatsApp-Kommunikation, auf die inzwischen oft als WhatsApp-Chat referiert wird, und der klassischen Chat-Kommunikation via Internet Relay Chat, indem er die für beide Kommunikationsformen charakteristische Tendenz zum Aufsplitten von Beiträgen im Sinne Beißwengers (2007) aufzeigt, mit welchem dem sogenannten Mühlenprinzip entgegengewirkt wird (vgl. auch Chunking bei Wyss/Hug 2016: 270 sowie Dürscheid 2016: 454-455). Ein solches Schreibverhalten wird laut Imo (2015a: 16) v.a. durch die äußerst geringen Kosten ermöglicht: Man bezahlt nicht jede einzelne Nachricht, sondern lediglich das verbrauchte Datenvolumen des mobilen Internets, wobei viele Nutzer auch über eine Internet-Flatrate verfügen, sodass keine zusätzlichen Kosten für mehr Nachrichten anfallen. Es sei hier auch angemerkt, dass WhatsApp erst seit 2016 eine kostenfreie App geworden ist. Davor wurde nach dem Ablauf des ersten Jahres eine Gebühr in Höhe von 0,99 Dollar pro Jahr erhoben.

Eine weitere Motivation für das Verschicken einer Nachricht pro sprachliche Handlung sieht Imo (2015a: 16-17) in der besonderen Darstellung der WhatsApp-Nachrichten, die nicht wie SMS-Nachrichten auf alten Handys jeweils einzeln auf dem Bildschirm angezeigt werden, sondern als „fortlaufende Dialoge“83 visualisiert werden (Imo 2015a: 27; vgl. auch Dürscheid/Frick 2014: 172 sowie Dürscheid 2016: 449-454). Eine solche Darstellung wirkt sich laut Wyss/Hug (2016: 264-265, 269, 271) folgendermaßen auf die WhatsApp-Kommunikation aus: Beim ,Kampf' um den conversational floor wird nicht nur dem zeitlichen (,Wer schickt seinen Beitrag als Erster ab?'), sondern auch dem räumlichen Aspekt (,Wessen Beiträge nehmen mehr Platz auf dem Bildschirm ein?’) eine große Bedeutung zugemessen (vgl. dazu die sogenannte Pushing-Strategie im Sinne von Wyss/Hug 2016: 270-272):

Einzeln verschickte Turns lassen sich vielleicht angenehmer und rascher rezipieren, aber weit wichtiger ist, dass der Produzent durch die Zergliederung eine visuelle lokale Dominanz erreicht: Seine Beiträge dominieren den Raum, indem sie einen Großteil der Projektionsfläche besetzen und Relevanz behaupten. Auf diese Weise wird dem Leser zudem

83 Vgl. auch König (2015a: 103): „Während die SchreiberInnen in der SMS-Kommunikation auf der sprachlichen Oberfläche durch Anreden Dialogeinheiten in der Regel als distinkt abgrenzen, ist eine solche klare Untergliederung für die untersuchten WhatsApp-Nachrichtenstränge nicht mehr ohne Weiteres feststellbar. Vielmehr sind die Interaktionen auch über thematische und zeitliche Brüche hinweg als kontinuierlich andauernder Austausch gerahmt“. 
signalisiert, dass hier jemand den Gesprächsfloor hält und das Rederecht nicht abgeben möchte. (Wyss/Hug 2016: 271)

Das Verschicken kurzer WhatsApp-Nachrichten ermöglicht einen relativ schnellen Nachrichtenaustausch, in dessen Rahmen wenige Sekunden zwischen einzelnen Beiträgen liegen können, sodass die über die Chat-Funktion verlaufende schriftbasierte Kommunikation zwischen zwei oder mehreren Personen in Anlehnung an Dürscheid (2005) als quasi-synchron eingeordnet werden kann (vgl. Dürscheid/Frick 2014: 166-167; Imo 2015a: 7). Dabei weist Dürscheid (2016: 456) aber zurecht darauf hin, dass die Quasi-Synchronie „als skalarer Begriff“ konzeptualisiert werden muss und dass ihr Grad im Laufe eines Dialogs je nach dem zeitlichen Abstand zwischen einzelnen Nachrichten variieren kann. Eine ähnliche Überlegung findet sich auch bei König (2015a: 90, 100-101), die allerdings das Kontinuum der auf die WhatsApp-Kommunikation potenziell zutreffenden zeitlichen Dimension noch breiter - von quasi-synchron bis zu asynchron - fasst. ${ }^{84}$ Der ohnehin hohe „Interaktionsgrad“85 (Dürscheid/Frick 2014: 170-172) wird zusätzlich dadurch verstärkt, dass zum einen angezeigt werden kann, dass der Kommunikationspartner gerade eine Nachricht verfasst (durch die unter dem Namen des Interaktionspartners angezeigte Meldung, z.B. Irina schreibt...). Zum anderen aber - falls diese Funktion nicht absichtlich deaktiviert wurde - wird durch zwei blaue Häkchen in der rechten unteren Ecke einer Nachrichtenblase markiert, dass die Nachricht vom Rezipienten (zumindest) geöffnet und ggf. auch gelesen wurde (vgl. Dürscheid/Frick 2014: 167; Imo 2015a: 7; König 2015a: 90).

Als eine der Auswirkungen des hohen Interaktivitätsgrads der WhatsAppKommunikation sowie der damit einhergehenden Tendenz zum Verschicken mehrerer kurzer Textnachrichten sehen Dürscheid/Frick (2014: 170) das Entstehen von „Überlappungen und Inkohärenzen im Textverlauf“ (vgl. auch Wyss/Hug 2016: 268-269; zu ähnlichen Phänomenen in der Kommunikation im spielbegleitenden Chat vgl. Collister 2008: 65-66). In diesem Zusammenhang

84 Sprachnachrichten dagegen werden von König/Hector (2017: 12-13) eher als asynchrone Kommunikation angesehen, da diese ,in einem doppelten Sinn verzögert“ sind, und zwar zum einen durch das Aufnehmen einer Sprachnachricht und zum anderen durch das Abspielen dieser Nachricht bei deren Rezeption.

85 Die Angemessenheit der Verwendung des Terminus Interaktion in Bezug auf die Analyse der WhatsApp-Kommunikation wird von Dürscheid (2016) diskutiert, während Dürscheid (2016) sowie Wyss/Hug (2016) auf das Ausbleiben der Wahrnehmungswahrnehmung in der informellen Schriftlichkeit und die sich in diesem Zusammenhang für die WhatsAppKommunikation ergebenden Folgen eingehen. 
können beispielsweise in zukünftigen Untersuchungen die Strategien in den Blick genommen werden, mit denen die eindeutige Zuordnung zwischen dem zweiten Teil einer Paarsequenz und einem zurückliegenden ersten Paarteil gewährleistet wird. Eine Verbindung zwischen zwei Nachrichten einer Paarsequenz kann u.a. hergestellt werden, indem der erste Paarteil in Form eines Zitats in den Text des zweiten Paarteils integriert wird. Diese bereits aus der Foren-Kommunikation vertraute Strategie ist auf dem in (87) abgebildeten Screenshot (vgl. \#6) zu sehen:

(87) Auszug aus den für die russische Version der MoCoDa $2^{86}$ erhobenen Daten (Zitieren einer WhatsApp-Nachricht in einem reaktiven Posting):

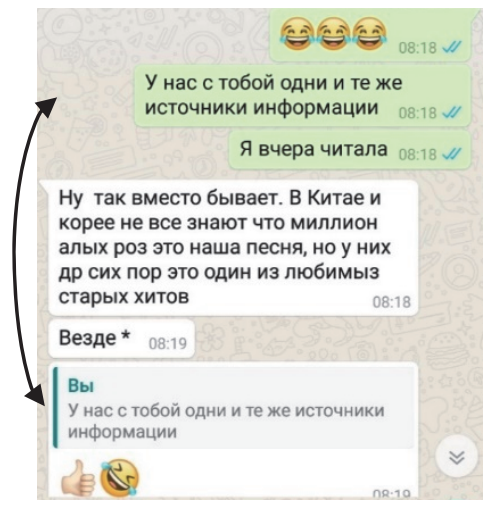

$1 \mathrm{~B}: 03$

2 B: Wir haben die gleichen Informationsquellen

3 B: Ich habe gestern darüber gelesen

4 A: Das passiert ja anstatt. In China und Korea wissen auch nicht alle, dass „Eine Million roter Rosen" unser Lied ist, aber es gilt bei ihnen bis heute als einer der beliebtesten alten Hits

5 A: Überall *

6 A: Du

Wir haben die gleichen Informationsquellen 낭

Im Laufe der Besprechung der Herkunft eines Songs greifen die beiden Schreiberinnen auf den entsprechenden Wikipedia-Eintrag zurück und legen ihre Quelle bei der Diskussion offen, sodass eine Art Überlappung entsteht, auf die Schreiberin $B$ (Nachrichten rechts) mit einem iterierten Emoji reagiert, das in dem Fall eine evaluierend-kommentierende Funktion erfüllt (vgl. Pappert 2017). Anschließend äußert sie sich zur entstandenen Überlappung auch verbal (\#2) und teilt mit, dass sie den Wikipedia-Artikel am vorigen Tag gelesen hat (\#3). Nachricht \#4 bezieht sich auf die laufende Song-Besprechung, während in \#5

86 Neben der deutschen Version der Datenbank MoCoDa 2 (https://db.mocoda2.de), die die MoCoDa im Jahre 2018 abgelöst hat, wurde 2020 eine separate Seite für die russische Version der MoCoDa 2 eingerichtet, die sich momentan in der Testphase befindet. Bis zur Fertigstellung der russischen Version der MoCoDa 2 werden WhatsApp-Verläufe in Form von Screenshots und Chat-Logs erhoben, die anschließend in die Datenbank eingepflegt werden. Einige dieser Screenshots werden in der vorliegenden Arbeit herangezogen, um einzelne Phänomene zu belegen, die sich anhand der Daten aus der MoCoDa nicht veranschaulichen lassen. 
ein Fehler aus \#4 korrigiert wird („вместо“ ,anstatt“ wird durch „везде“ ,überall‘ ersetzt). Bei der sich daran anschließenden Kombination aus zwei Emojis, die eine evaluative Funktion (vgl. Pappert 2017) übernehmen, wird das Zitieren einer Nachricht eingesetzt, um die Zuordnung der mit den Emojis transportierten Bewertung zu ermöglichen. ${ }^{87}$ Die zitierte Nachricht (\#2) wird hellgrau hinterlegt und damit graphisch vom Rest der Antwortnachricht abgehoben. Der Autor der zitierten Nachricht wird explizit genannt: In der linken oberen Ecke erscheint entweder der Name des Interaktionspartners oder - beim Zitieren eigener Beiträge - das Personalpronomen $D u$ bzw. Bы (,Sie'). Eigene und fremde Nachrichten, die als Zitate wiederaufgenommen werden, können auch an anderen farblichen Markierungen erkannt werden: Die Schriftfarbe des Autorennamens sowie die Farbe der linken Randlinie des Zitats ist bei eigenen Nachrichten türkis und bei fremden lila. Die kommunikative Funktion des Zitierens einer Nachricht für den Reparaturmechanismus muss allerdings noch näher untersucht werden.

Einen weiteren wichtigen Gegenstand der WhatsApp-Forschung stellt der Emoji-Gebrauch dar, wobei v.a. einzelne kommunikative Funktionen von Emojis diskutiert und herausgearbeitet wurden (vgl. für das Deutsche Dürscheid/Siever 2017; Pappert 2017; Siebenhaar 2018; Beißwenger/Pappert 2019 sowie für das Russische Krylov 2017; Sergeeva/Dzyuba/Rybasova 2017). Nach der Einführung von Stickern, die im Jahr 2018 erfolgte, wäre deren Einbindung in die Interaktion via WhatsApp auch ein Thema für zukünftige Untersuchungen (vgl. Hayashi/Imo/Ogasarawa 2020). Darüber hinaus liegen Arbeiten zum Code-Switching in der WhatsApp-Kommunikation (vgl. Zagoricnik 2014), zu interaktionalen Funktionen von onymischen Anreden (vgl. Bauer 2016), graphischen Reparaturmarkern (vgl. Mostovaia 2018) sowie Fremdinitiierungen (vgl. Mostovaia 2021) vor.

Im Gegensatz zur WhatsApp-Kommunikation ist die Viber- sowie iMessage-Kommunikation aus linguistischer Perspektive kaum erforscht, was mit den geringen Nutzerzahlen für die jeweilige App erklärt werden kann (vgl. die Statistik am Anfang dieses Kapitels). Da WhatsApp mit einer Nutzerquote von über 80\% in Deutschland die mit Abstand meist genutzte App darstellt, ist es nicht verwunderlich, dass im deutschsprachigen Raum v.a. Arbeiten zur WhatsApp-Kommunikation entstehen, während die beiden Messenger Viber und

87 Es ist auffällig, dass auf der russischsprachigen Oberfläche von WhatsApp auf den Verfasser der zitierten Nachricht mit einem distanziert höflichen Personalpronomen $B ы$ (,Sie`) referiert wird, während in der deutschsprachigen Version an derselben Stelle das Pronomen $D u$ erscheint, wobei nicht klar ist, ob die Großschreibung dabei intendiert eingesetzt wird. 
iMessage dabei i.d.R. nur am Rande bei der Auflistung unterschiedlicher Messenger-Apps erwähnt werden (vgl. König/Bahlo 2014: 8; Imo 2015a: 5; Wyss/Hug 2016: 260). In der russischsprachigen linguistischen Forschung liegen bisher zwar generell relativ wenige Untersuchungen zur Kommunikation über mobile Messenger-Dienste vor, aber diese widmen sich neben der WhatsApp- auch der Viber-Kommunikation, was dadurch bedingt zu sein scheint, dass Viber mit $38 \%$ eine relativ hohe Nutzerquote in Russland aufweist. So befasst sich Minchuk (2018) anhand von Viber-Gruppenchats zwischen russischsprachigen Schreiberinnen aus Weißrussland, Norwegen und Russland mit idiosynkratischen Entlehnungen sowie dem Code-Switching zwischen dem Russischen und dem Weißrussischen. Orlova (2018) führt eine umfragebasierte Studie zum Nutzungsverhalten beim Gebrauch von Sprachnachrichten durch, die per WhatsApp oder Viber verschickt werden, gibt jedoch bei der Auswertung der Ergebnisse weder an, in wie vielen Fragebögen jeweils auf die eine bzw. die andere App eingegangen wurde, noch legt sie offen, ob Unterschiede im Umgang mit Sprachnachrichten in den beiden Messenger-Anwendungen festgestellt wurden. Somit wird implizit suggeriert, dass die Kommunikation über Sprachnachrichten in WhatsApp und Viberidentisch verläuft.

Eine ähnliche Tendenz zur gemeinsamen Analyse der Daten aus mehreren Messenger-Apps zeigt sich auch bei Goroshko/Zemlyakova (2017). Hierbei handelt es sich allerdings um eine Analyse von Text- und Sprachnachrichten, die via Viber sowie Telegram versandt wurden (über 500 Nachrichten, erhoben im März-April 2017). Angenommen, dass die bei Goroshko/Zemlyakova (2017) aufgeführten Merkmale gleichermaßen auf die Viber- und Telegram-Kommunikation zutreffen, lässt sich die Viber-Kommunikation in Anlehnung daran folgendermaßen beschreiben: Die Viber-Kommunikation ist dialogisch ausgerichtet, die Anzahl der Interaktionsteilnehmer kann von zwei bis 300 Personen variieren. Als Kommunikationsmedium dient sowohl ein Smartphone als auch ein Tablet und ein PC (55,1\% der Informanten gaben an, dass sie die Messenger-Apps auch auf ihren PCs installiert haben). Aus der zeitlichen Perspektive wird die Viber-Kommunikation aufgrund der i.d.R. geringen Reaktionszeit als synchron angesehen, wobei mit Dürscheid (2005) die Anordnung als quasi-synchron sinnvoller erscheinen mag. Es wird auch auf die Tatsache hingewiesen, dass via Viber - ähnlich wie via WhatsApp - nicht nur Textnachrichten, sondern Fotos, Bilder, Sticker, Videos, Sprachnachrichten etc. verschickt werden können. Aus diesem Grund wird Viber als „полиформатный мессенджер“ ( $\approx$,Messenger mit vielen Nachrichtenformaten“) bezeichnet. Zudem wird auch auf den konzeptionell mündlichen Charakter der Viber-Kommunikation eingegangen (vgl. „устно-письменный характер речи“ ,münd- 
lich-schriftlicher Charakter der Kommunikation'). In Bezug auf verbale und nonverbale Mittel gleicht der Sprachgebrauch in der Viber-Kommunikation laut Goroshko/Zemlyakova (2017) weitgehend dem in der SMS-Kommunikation (vgl. Kap. 4.1): Es werden u.a. solche Phänomene wie Ellipsen, Reduktionen, durchgehende Kleinschreibung, Majuskelschreibung als graphische Realisierung der emulierten Prosodie, häufiges Vorkommen von Interjektionen etc. genannt. Allerdings wird nicht auf Emojis eingegangen, sondern lediglich auf Emoticons, von denen :), ;), :( sowie deren reduzierte Formen ) und (als die frequentesten aufgeführt werden (vgl. Goroshko/Zemlyakova 2017: 98). Des Weiteren wurde zwar explizit angegeben, dass sich das Datenkorpus aus Text- und Sprachnachrichten zusammensetzt, dennoch werden keine Sprachnachrichten bei der Analyse aufgeführt.

Mit dem Emoji-Gebrauch aus der Perspektive der Genderforschung beschäftigt sich die Studie von Al Rousan/Remil (2018), die anhand von 2.616 ViberNachrichten von algerischen Studierenden sowohl qualitative als auch quantitative Divergenzen feststellen konnten, die einerseits psychologisch, andererseits aber auch kulturspezifisch begründet werden (zu Emojis und Gender in der deutschen WhatsApp-Kommunikation vgl. Fladrich/Imo 2020).

In Bezug auf die iMessage-Kommunikation konnte lediglich eine Studie gefunden werden, die sich mit der multimodalen Analyse des Gebrauchs von Stickern auseinandersetzt (vgl. Moschini 2018). Leider werden dabei nicht iMessage-Nachrichten aus authentischen Verläufen untersucht, sondern in erster Linie Beispiele aus Werbespots, in denen demonstriert wird, wie diese Sticker eingesetzt werden könnten.

Mit Blick auf den aktuellen Forschungsstand sind Untersuchungen zur Viber- und iMessage-Kommunikation im Vergleich zu denen zur WhatsApp-Kommunikation deutlich unterrepräsentiert. Dabei wird in den wenigen derzeit vorliegenden Arbeiten, in denen Viber oder iMessage nur erwähnt oder auch etwas ausführlicher analysiert werden, nicht darauf eingegangen, ob und inwiefern sich die Kommunikation in unterschiedlichen Messenger voneinander unterscheidet, sondern es wird im Grunde genommen suggeriert, dass es die Messenger-Kommunikation gibt, die lediglich über unterschiedliche Apps realisiert wird. Die Frage, ob diese Annahme der Realität entspricht oder ob sich die heutzutage allerdings recht subtilen Unterschiede in der technischen Funktionalität einzelner Messenger (wie z.B. die sogenannte Tapback-Funktion ${ }^{88}$ bei

88 Die Tapback-Funktion ist vergleichbar mit dem Like-Button bei Facebook. Bei dieser Funktion werden sechs verschiedene evaluative Symbole bereitgestellt (ein Herz, Daumen hoch, Daumen runter, haha, zwei Ausrufezeichen und ein Fragezeichen), mit denen man aber nicht 
iMessage) doch auf das Sprachverhalten ihrer Nutzer auswirken, lässt sich leider im Rahmen dieser Arbeit nicht beantworten, da das Untersuchungskorpus einen zu geringen Anteil an Viber- und iMessage-Dialogen aufweist. Von 607 russischen Kurznachrichtendialogen sind lediglich 17 über Viber und 18 über iMessage erfolgt, während in 2.206 deutschen Dialogen nur 20 iMessage-Dialoge und keine Viber-Dialoge enthalten sind (vgl. Kap. 5).

auf Pinnwandeinträge, sondern auf innerhalb von Chat-Verläufen verschickte Nachrichten reagiert. Das ausgewählte Symbol erscheint nach dem Abschicken nicht als selbstständige Antwortnachricht, sondern wird an die Nachricht, auf die es sich bezieht, ,angepinnt‘. 